\title{
Global and local contributions to surface curva- ture of healthy corneas
}

\author{
Alan Rubin* and Solani David Mathebula ${ }^{\dagger}$ \\ *Department of Optometry, University of Johannesburg, PO Box 524, Auckland Park, 2006 South \\ Africa
}

${ }^{\dagger}$ Department of Optometry, University of Limpopo, Private Bag X1106, Sovenga, 0727 South Africa

<arubin@uj.ac.za>

$<$ solani.mathebula@ul.ac.za>

Received 19 July 2012; revised version accepted 2 November 2012

\begin{abstract}
This paper demonstrates for several healthy eyes the application of a simple model to understanding local and global contributions to short-term variation in anterior and posterior corneal curvature. Multiple axial anterior and posterior corneal radii and central corneal thicknesses for the right eyes of 10 young subjects were determined over time using a rotating Scheimpflug camera (Oculus Pentacam). The axial radii were transformed to corneal powers, and also to curvatures that were referred to a mid-corneal surface such that local and global contributions to short-term variation could be analyzed quantitatively.

When variation of the anterior and posterior corneal surfaces of several healthy eyes are studied in terms of curvatures (rather than powers) it is the posterior surfaces that are more variable with the global or macroscopic rather than local effects dominating. (Harris and Gillan found the same for an eye with mild keratoconus.) This finding is opposite to that when variation is considered in terms of dioptric power where the anterior corneal surface usually appears more variable. Possible reasons for this finding includes firstly that the posterior corneal surface has to be measured through the air-tear interface and anterior corneal surface,
\end{abstract}

and thus some uncertainty in measurements of the posterior surface may relate to this limitation. Secondly, no attempt was made here to mathematically align the multiple surfaces as determined per eye and thus we cannot be certain that precisely the same central corneal region was measured each time.

Investigators need to carefully consider whether they are more interested in the optical or physical nature of variation in surfaces such as the cornea since studies of the optical effects require the analysis to be performed in terms of dioptric powers and symmetric dioptric power space whereas studies of physical variation in the topography of the cornea and the possible reasons for such variability require the application of surface curvatures in surface curvature space. This paper describes the application and significance of both methods to facilitate understanding of short-term variation of the human cornea. It does not, however, attempt to make any definite claims as to what factors (see above) may be major contributors to such variability, and this complicated but interesting research issue requires further clarification. ( $S$ Afr Optom 2012 71(4) 146-158)

Key words: Dioptric power, curvature, cornea, variance, vector spaces, multivariate statistics. 


\section{Introduction}

The cornea is an extremely complicated and dynamic optical structure of the human eye. Further complicating any study of the human cornea is the fact that it is difficult to truly separate the cornea from its pre-corneal tear film and this can markedly complicate in vivo measurements of parameters such as anterior corneal power or thickness. The air-tear interface is, however, a critical one in terms of the optical properties of the anterior part of the eye and its interactions with light. At, perhaps, the simplest level we can model the optical system of the cornea as a single thin lens in air, and of uniform power and no thickness. A slightly more sophisticated approach would be to consider the cornea as a thick lens in air, having both anterior and posterior surfaces of constant power separated by uniform thickness. In reality the cornea varies both in terms of the separation between its surfaces and also in the surface powers and shape or topographies. Such variation is not only in relation to temporal but also to spatial factors or geometric location. Even the refractive index of the cornea is similarly not constant and varies in a complicated fashion in relation to many variables such as time of day and state of health ${ }^{1}$. Many attempts have been made, and several methods ${ }^{2}$ are available, to more completely understand some of these isolated aspects of corneal physiology and structure such as variation in central power of both healthy ${ }^{3-5}$ and, for example, keratoconic corneas ${ }^{6,7}$, and diurnal variation in corneal shape and thickness ${ }^{8}$. Fortunately we have modern methods such as corneal topography ${ }^{2}$, wavefront aberrometry and optical coherence tomography that greatly assist us in some of these endeavors ${ }^{2}$. But, as Twa et al ${ }^{9}$ emphasize, the interpretation of the cornea via methods such as videokeratography remains a major challenge and they suggest the use of automated models to classify corneal shape.

Yet another important issue, when determining multiple measurements over the short-term, is that clinical instruments using Scheimpflug cameras do not necessarily measure exactly the same regions of the eye. So, although we probably can assume that roughly the same regions are measured, some measurement uncertainty or instrument noise may occur and this issue will be reconsidered later in this paper.
This paper will focus on a simple model proposed by Harris ${ }^{4}$ to understand variation in anterior and posterior corneal curvatures. This model of corneal curvature has previously been applied to study a single keratoconic cornea ${ }^{6,7}$ but here it will be considered in relation to results for ten healthy corneas instead. Gillan ${ }^{6}$ found when using dioptric powers that the anterior, rather than posterior, surface of a keratoconic cornea apparently varied to a greater extent over a short period but when the surface curvatures were instead used the opposite was true ${ }^{7}$. This suggests the need to be careful when attempting to understand possible sources of variation in different ocular variables such as corneal shape (a physical or geometric property of the cornea) and its dioptric power (an optical property $)^{4,6,7}$. The measurements used here were obtained as part of data acquired by Mathebula in a study of the linear optical characteristics of the anterior eye ${ }^{5}$. In this work ${ }^{5}$, Mathebula describes the cornea in terms of ray transferences ( $4 \times 4$ matrices) where the surface powers and axial corneal thickness are important for studying variation in the complete optical character of the cornea. When considering variation of the cornea, some investigators such as Gillan ${ }^{6}$ and others ${ }^{3,}{ }^{8-18}$ have concentrated their attention on anterior, or less commonly posterior, surface dioptric powers (typically without the use of transferences) or sometimes on corneal radii or curvatures. Some authors have considered both corneal powers and curvatures in their papers, often in reference to a multitude of variables or factors such as, for example, astigmatism ${ }^{12,13}$ or orthokeratology ${ }^{17}$. In this paper we mainly use the surface curvatures that, we believe, are more relevant to making proper sense of the basic or fundamental causes of variation in the physical shape of the corneal surfaces.

A concise explanation of some of the theoretical concepts and equations of the model will be included but see Harris ${ }^{4}$ for full details. In this simple model the cornea (of refractive index, $n_{2}$ ) is regarded as having two surfaces, namely $\mathrm{S}_{1}$ and $\mathrm{S}_{2}$, separated by central thickness $t$. Anterior to $\mathrm{S}_{1}$ and posterior to $\mathrm{S}_{2}$, the refractive indices are $n_{2}$ and $n_{3}$ respectively and would usually be the refractive indices of air and aqueous humor. We then can define a surface $\left(\mathrm{S}_{\mathrm{m}}\right)$ in the middle of the cornea that is separated from each of $\mathrm{S}_{1}$ and $\mathrm{S}_{2}$ by distance, $t / 2$. Surface curvature, $\mathcal{K}$ is 
related to symmetric dioptric power, $\mathbf{F}$, by the simple linear equation as defined by Harris ${ }^{4}$ :

$\mathbf{F}=\mathcal{K} \Delta n$.

Both $\mathbf{F}$ and $\mathcal{K}$ are $2 \times 2$ symmetric matrices, $\Delta n$ is the refractive index after the surface minus the index before it, and Equation 1 can be expanded as:

$\left(\begin{array}{ll}f_{11} & f_{12} \\ f_{21} & f_{22}\end{array}\right)=\left(\begin{array}{ll}\kappa_{11} & \kappa_{12} \\ \kappa_{21} & \kappa_{22}\end{array}\right) \Delta n$.

The left side of the equation is simply the dioptric power matrix (see Blendowske ${ }^{19}$ concerning Fick's contributions and Long ${ }^{20}$ ) while the diagonal entries in $\mathcal{K}$ are curvatures in the reference (taken as horizontal here) and corresponding orthogonal meridians respectively. The off-diagonal entries $\left(\mathcal{K}_{21}=\mathcal{K}_{12}\right.$ where the matrix is symmetric) are measures of the torsional curvature in the reference meridian. The entries in $\mathcal{K}$ can be used to determine coefficients of curvature $\left(\mathcal{K}_{\mathrm{I}}, \mathcal{K}_{\mathrm{J}}\right.$ and $\left.\mathcal{K}_{\mathrm{K}}\right)$ that are useful for graphical representations of variation in curvature of the cornea (see Figure 2) and these curvature components will be explained in more detail later.

Since this simple model of the cornea has both anterior and posterior surfaces we have two axial or central dioptric powers $\mathbf{F}_{1}$ and $\mathbf{F}_{2}$ where the subscripts 1 and 2 refer to the anterior and posterior surfaces in turn. We can determine these powers using, for example, Scheimpflug photography. (We need to convert from clinical notation in terms of sphere and cylinder powers, and cylinder axis to dioptric power matrices ${ }^{19,20}$.) Their corresponding anterior and posterior surface curvatures (Equation 1) are then $\mathcal{K}_{1}$ and $\mathcal{K}_{2}$ respectively. We can now consider that the anterior or posterior corneal surfaces possibly could have local and global changes in curvature. For instance, local or perhaps microscopic effects may be due to tiny ripples in one or both surfaces whereas a global or macroscopic change might be induced by closure of the eyelid during blinking6, 7 . To better understand the relative contributions to variance of corneal curvature we need to separate the local and global contributions or effects from each surface and preferably refer them to a mid-corneal surface $\left(S_{m}\right.$ as described above) since corneal axial thickness could be an important variable 6,7 . The global effects are dependent on the conformity of curvature of the anterior and posterior surfaces whereas the local effects are related to their non-conformity or differences. Harris ${ }^{4}$ calls the former $\mathcal{K}_{m}$ and the latter $\mathcal{K}_{d}$ and Equations 3 and 4 below (originally Equations 17 and 18 in reference 4) are measures of the global and local contributions referenced to the corneal mid-surface:

$$
\begin{aligned}
& \mathcal{K}_{m}=\frac{1}{2}\left(\mathcal{K}_{2 m}+\mathcal{K}_{1 m}\right) \\
& \mathcal{K}_{d 2}=\frac{1}{2}\left(\mathcal{K}_{2 m}-\mathcal{K}_{1 m}\right)
\end{aligned}
$$

where 4

$$
\mathcal{K}_{1 m}=\left(\mathcal{K}_{1}^{-1}-\frac{t}{2} \mathbf{I}\right)^{-1}
$$

and

$$
\mathcal{K}_{2 m}=\left(\mathcal{K}_{2}^{-1}+\frac{t}{2} \mathbf{I}\right)^{-1} \text {. }
$$

The matrix $\mathbf{I}$ is the $2 \times 2$ identity and $t$ is axial corneal thickness. In Equations 3 and 4 the subscripts 1 and 2 refer to the anterior and posterior surfaces in turn. In Equation 4 the local effects at the mid-surface are defined relative to the posterior or second surface but instead could be done relative to the first surface ${ }^{4}$ using $\mathcal{K}_{d 1}=\frac{1}{2}\left(\mathcal{K}_{1 m}-\mathcal{K}_{2 m}\right)$.

In summary, this paper will investigate global $\left(\mathcal{K}_{m}\right)$ and local $\left(\mathcal{K}_{d 2}\right)$ curvature contributions using a simple model $^{4}$ where measurements of variance in corneal curvature are referenced to the mid-corneal surfaces of each of 10 right eyes.

\section{Method}

\section{Subjects and Procedures}

Data here was obtained from the study 5 approved by the Higher Degrees and Ethics Committees of the Faculty of Health Sciences of the University of Johannesburg and the investigation was carried out with due regard to the tenets of the Declaration of Helsinki. Participants were fully advised about the nature and aims of the study and they provided their informed consent before inclusion. Exclusion criteria were any ocular disease or surgery and current contact lens wear. Ten healthy young subjects (nine females and one male) of age range 21-34 years were selected and Scheimpflug photography was performed repeatedly $(N=43)$ on the right eyes of each 
individual using the Oculus Pentacam (model 70700). The reason for 43 scans per subject was to allow one to three scans to be possibly removed should they be regarded as outliers. After each of these 43 scans (where the instrument acquired 25 cross-sectional images of the corneal and anterior segment in less than two seconds per scan) the subject was requested to remove their head from the instrument while the instrument processed and saved the results. The time period over which these measurements were obtained was about 50 minutes per subject as our interests were in studying short-term variation in various parameters such as corneal thickness and anterior and posterior corneal curvature and power. Some results (but not including for corneal curvatures) from this study 5 have been reported elsewhere ${ }^{21-23}$. Biomicroscopy, ophthalmoscopy and subjective refractions and visual acuities were performed and all subjects had best compensated visual acuity of $20 / 20$ or better in their right eyes 5 .

\section{Data analysis}

For each subject the anterior and posterior corneal principal radii of curvatures from the Pentacam were transformed to dioptric power matrices. Anterior corneal curvatures were also determined using Equation 1 and refractive indices of the cornea $(n=1.376)$ and air $(n$ $=1)$. Posterior corneal curvatures were calculated using, again Equation 1, but with refractive indices of 1.376 and 1.336 for the cornea and aqueous respectively. The curvatures for each subject were used to determine their coefficients of curvature, $\mathcal{K}_{\mathrm{I}}, \mathcal{K}_{\mathrm{J}}$ and $\mathcal{K}_{\mathrm{K}}$ namely the spherical, ortho-antispherical and oblique antispherical coefficients respectively. For any $2 \times 2$ matrix $\mathcal{K}$ these coefficients are determined using the three equations ${ }^{4}$

$$
\begin{aligned}
& \mathcal{K}_{\mathrm{I}}=\frac{1}{2}\left(\kappa_{11}+\kappa_{22}\right), \\
& \mathcal{K}_{\mathrm{J}}=\frac{1}{2}\left(\kappa_{11}-\kappa_{22}\right), \\
& \mathcal{K}_{\mathrm{K}}=\kappa_{12}
\end{aligned}
$$

and the curvature components ${ }^{4}$ are $\mathcal{K}_{\mathrm{I}}=\mathcal{K}_{\mathrm{I}} \mathbf{I}, \mathcal{K}_{\mathrm{J}}=$ $\mathcal{K}_{\mathrm{J}} \mathbf{J}$ and $\mathcal{K}_{\mathrm{K}}=\mathcal{K}_{\mathrm{K}} \mathbf{K}$ where $\mathbf{I}=\left(\begin{array}{ll}1 & 0 \\ 0 & 1\end{array}\right), \mathbf{J}=\left(\begin{array}{cc}1 & 0 \\ 0 & -1\end{array}\right)$ and $\mathbf{K}=\left(\begin{array}{ll}0 & 1 \\ 1 & 0\end{array}\right)$
Using these curvatures, and the corresponding central corneal thicknesses from the Pentacam, Equations 3 and 4 were applied to determine for each subject the global and local contributions to variation in corneal curvature. A simple numerical example is included in the appendix to demonstrate the process for a single anterior and posterior corneal power and its corresponding central corneal thickness.

Further analysis of the curvature components with stereo-pairs of surface curvature space ${ }^{4,6,7}$ were done using software by Harris and Malan and modified by Rubin.

\section{Results}

One of the ten subjects was randomly chosen to illustrate typical results as obtained and Figure la indicates variation of corneal powers (from keratometric measurements obtained with the Pentacam) for the anterior and posterior corneal surfaces of the right eye of Subject 8 . The three axes of the stereo-pair are the stigmatic, ortho-antistigmatic and oblique antistigmatic axes and have labels 50I, $50 \mathrm{~J}$ and $50 \mathrm{~K}$ respectively. (Although this is really a graphical representation of part of an infinite 3-dimensional space of symmetric dioptric power matrices, one can also think of it in terms of coordinate axes of $50 \mathrm{M}, 50 \mathrm{~J}_{0}$ and $50 \mathrm{~J}_{45}$ where power vectors ${ }^{24}$ are instead used to indicate the corneal powers.) The scale (a tick interval of $10 \mathrm{D}$ or axis length of $50 \mathrm{D}$ in clinical terms) and origin, $\mathbf{O} \mathrm{D}$ were chosen so that the measurements for both corneal surfaces could be provided on a single stereo-pair of symmetric dioptric power space.

Two small black dots are seen in Figure 1a, one slightly below the origin being the keratometric powers for the posterior corneal surface and the other being near the label 50I being the powers for the anterior corneal surface. In (b) and (c) of Figure 1 the scale and origin are changed to a tick interval of 0.25 $\mathrm{D}$ and the sample mean applicable and we can see that each of the black dots in Figure 1a is actually a cluster of measurements $(N=43)$ with the powers for the anterior corneal surface (Figure $1 b$ ) varying to a slightly greater extent. That is, keratometric measurements appear in this subject to be more variable for the anterior rather than posterior corneal surface and this was similar to what Gillan found for the variation in corneal powers of his keratoconic 
subject $^{6}$. In Table 1 some results from Harris and Gillan $^{7}$ are provided and these findings will be compared with that for the subjects concerned here. a)

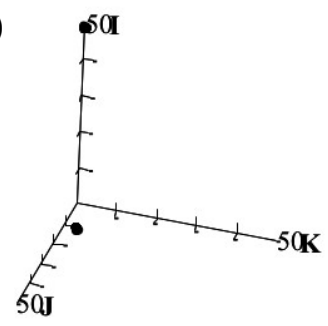

b)

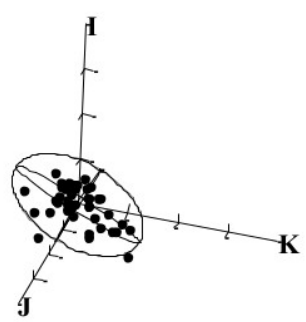

c)

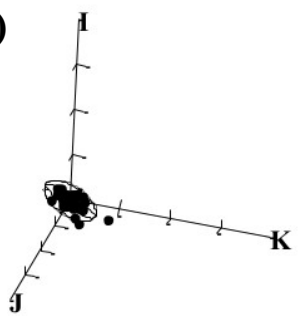

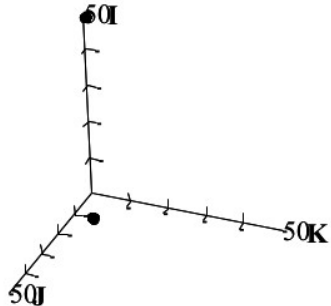
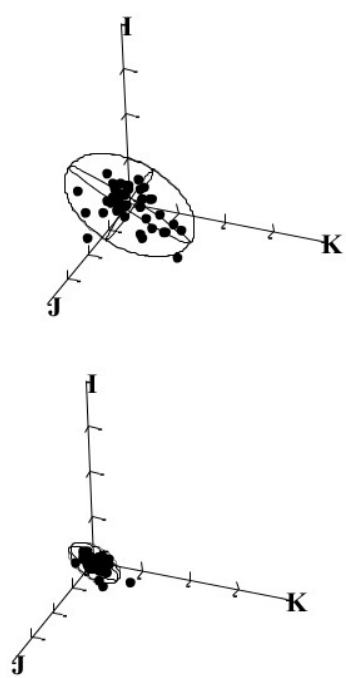

Figure 1. a) Stereo-pair scatter plot of variation in dioptric powers of the anterior and posterior corneal surfaces of Subject 8 are indicated in symmetric dioptric power space. Two small clusters of black dots are noted; one just below the origin (the power $\mathbf{O}$ D) and the other near the label 50I (for the stigmatic axis). Surfaces of constant probability density are also included for both samples but are not obvious at the scale used. The cluster near the origin represents short-term variation in corneal power of the posterior surface of this eye. b) The scale and origin are different from that in (a) enabling the variation in corneal powers of the anterior corneal surface of Subject 8 to become more easily visible. This is the same cluster as the one near the label 50I in (a). The origin is the sample mean and the tick interval is $0.25 \mathrm{D}$. The $95 \%$ surface of constant probability density has its longest principal diameter roughly parallel to the antistigmatic plane (that includes the antistigmatic axes, $F_{\mathrm{J}} \mathbf{J}$ and $\left.F_{\mathrm{K}} \mathbf{K}\right)$. c) Variation in corneal powers for the posterior corneal surface of Subject 8, with the corresponding 95\% surface of constant probability density is represented. This is the same cluster as the one near the origin $(\mathbf{O} \mathrm{D}$, in $(\mathbf{a})$, but here the origin is the sample mean and the tick interval is $0.25 \mathrm{D}$. The volume of the ellipsoid is smaller and measurements are more tightly clustered (than for the ellipsoid in (b)) showing that the anterior power of the cornea is more variable than that for the posterior power.
Table 1. Means and variance-covariance matrices (S) for a single keratoconic eye* from Harris and Gillan ${ }^{7}$ are included below. The $2 \times 2$ and $3 \times 3$ matrices below are all symmetric and thus only upper-triangular entries are included. For this keratoconic eye, the vectorized mean global and local effects in the corneal mid-surface are (141.5 7.3 148.8) $)^{\prime} \mathrm{D}$ and $(6.5$ $2.611 .2)^{\prime} \mathrm{D}$ respectively (see Table 2 for vectorized mean global and local effects for 10 healthy corneas from our study instead). Similarly, for this keratoconic eye $\mathrm{e}^{7}$ the global and local vectorized variance-covariances referred to the corneal midsurface are $\mathrm{D}^{2}$ and $\mathrm{D}^{2}$ respectively. In Table 2 typical global and local variances and covariances for healthy corneas of 10 eyes from our study are provided. In terms of curvature the posterior cornea is more variable than the anterior cornea. That is, the variances (or diagonal entries in the variance-covariance matrix) are larger for the posterior rather than anterior corneal surface. The global effects dominate with the ortho-spherical variance $\left(0.66 \mathrm{D}^{2}\right)$ greater than the other variances $(0.29$ and $0.19 \mathrm{D}^{2}$ ), probably relating to the effects of the eyelids upon the cornea during blinking in this keratoconic eye (with a possibly weakened or thinned cornea). The local contribution to variation in surface curvature is smaller than the global contribution as evidenced by their means. The variances $(0.21,0.25$ and 0.29 $\mathrm{D}^{2}$ ) for the local effects are similar thus indicating essentially random variation in surface curvatures that is not unexpected for small-scale or local effects. That is, variation is similar for all directions of surface curvature space and this is unlike that found for the global effects where variation was greater roughly along the ortho-antispherical or $\mathcal{K}_{\mathrm{J}} \mathbf{J}$ - axis. Mostly covariances in the various $3 \times 3$ matrices below are small or nearly zero indicating little evidence for linear relationships between the variances within each of the matrices concerned.

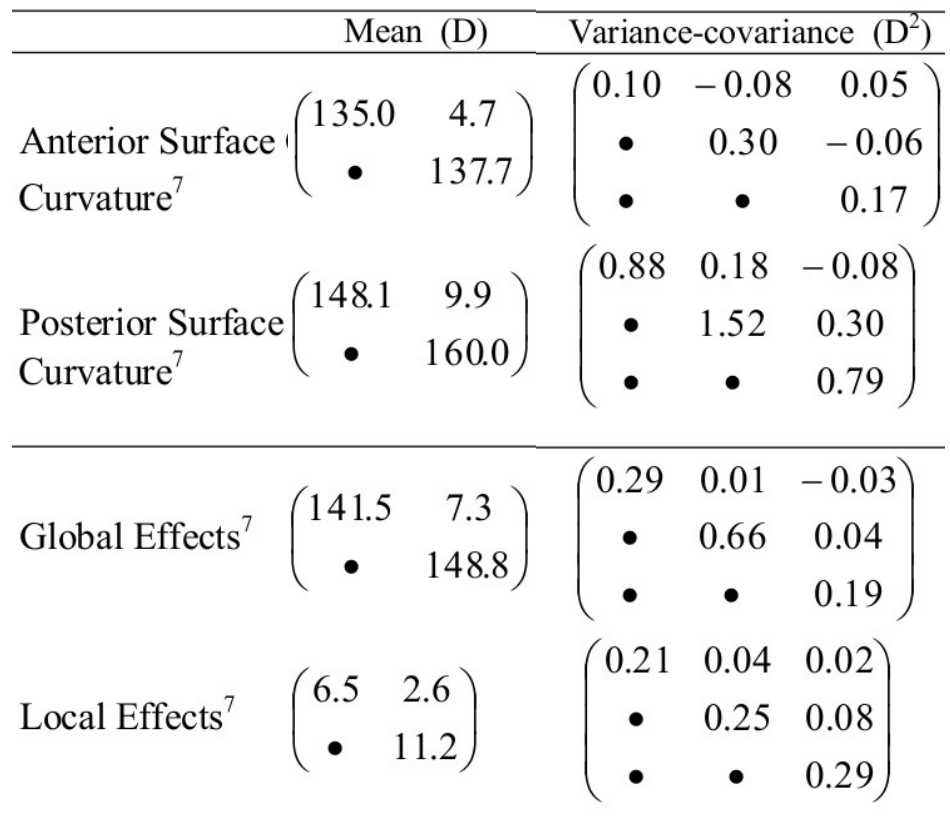

*All results in this table are from reference 7 
a)
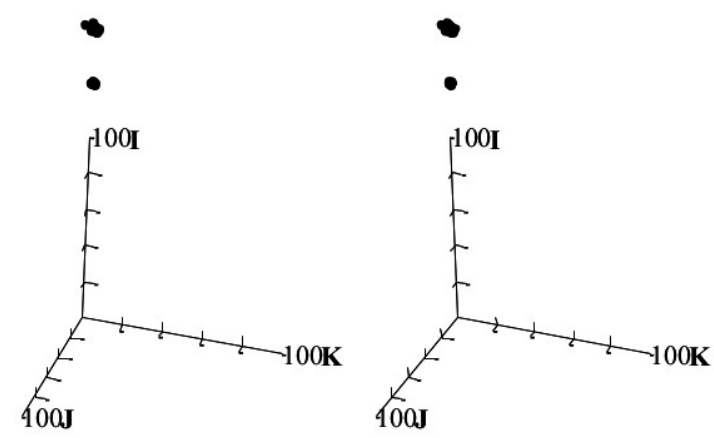

b)
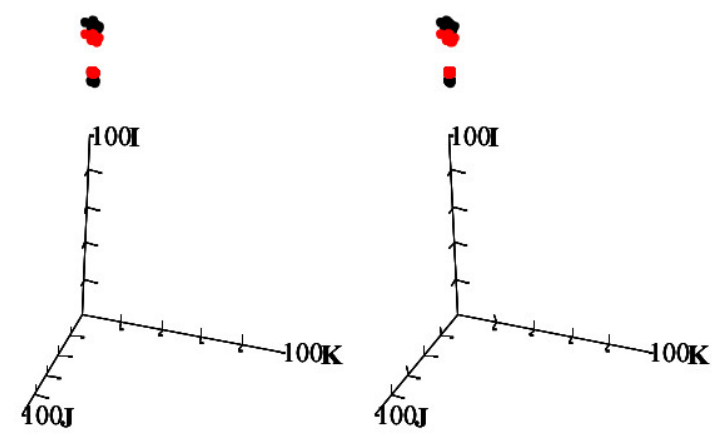

c)
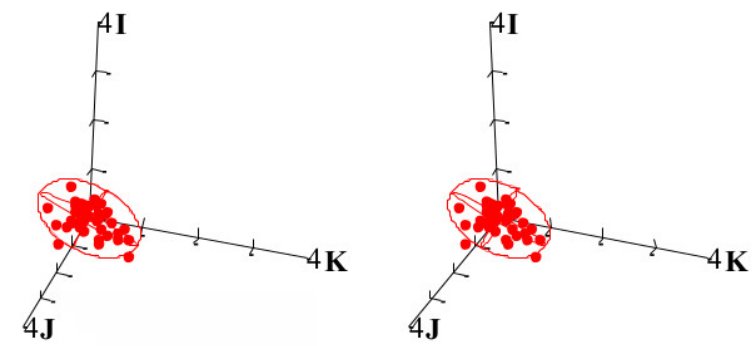

d)

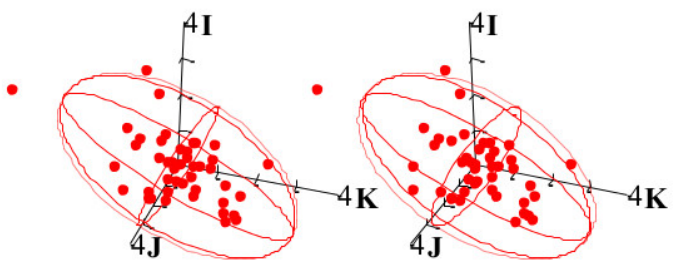

e)

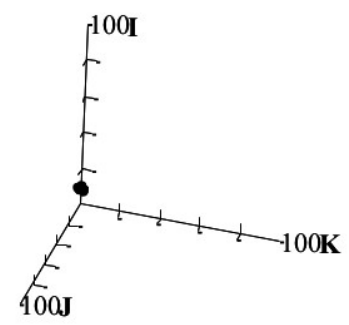

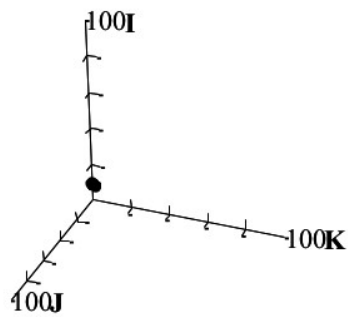

f)
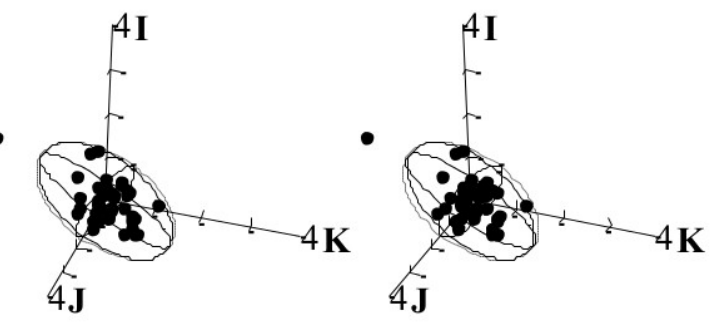

g)
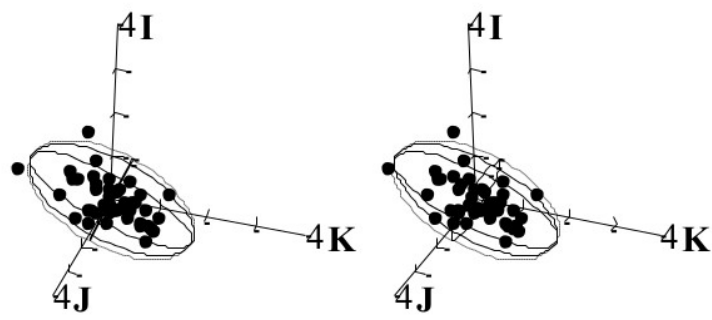

Figure 2. a) Stereo-pair plots of variation in curvature of the anterior and posterior corneal surfaces of Subject 8 are shown in surface curvature space. In the upper stereo-pair two small clusters of black dots are noted, just above the label 100I. Surfaces of constant probability density are also included for both samples but are not obvious at the scale used. The upper cluster that is slightly more variable is for the curvatures of the posterior corneal surface of this eye. The origin is the null curvature matrix, $\mathbf{O}$ D. After transformation of these measurements to the mid-corneal plane the clusters (in red) seen in the lower stereopair are slightly shifted in value. b) The scale and origin are changed so that the variation in anterior surface curvature (the lower red cluster in (a) in the mid-corneal plane and the $95 \%$ ellipsoid are more obvious. The origin is the sample mean (that is, the average anterior surface curvature) and the tick interval is $1 \mathrm{D}$. c) The scale and origin are such that the variation in posterior surface curvature (the upper red cluster in (a) in the mid-corneal plane and the $95 \%$ ellipsoid are more obvious. The origin is the sample mean (that is, the average posterior surface curvature) and the tick interval is $1 \mathrm{D}$. Unlike for the powers, in terms of curvature it is the posterior corneal surface that exhibits greater variation, that is, the ellipsoid is larger in (c) than in (b). d) Stereo-pair plot of variation in curvature of the anterior and posterior corneal surfaces of Subject 8 are shown in surface curvature space after reference to the mid-corneal plane and determining the local (indicated via the cluster of dots near the origin) and global effects (the cluster of dots above the label 100I). e) After modifying the scale and origin the local effects (the measurements of the cluster near the origin in (d) are indicated with the corresponding $95 \%$ surface of constant probability density. The centroid of the ellipsoid and here the origin also is the mean local effect and the tick interval is $1 \mathrm{D}$. f) After modifying the scale and origin, the global effects (the measurements of the cluster above the label 100I in (d) are indicated with the corresponding 95\% surface of constant probability density. The origin (and the centroid of the ellipsoid) is the mean global effect and the tick interval is $1 \mathrm{D}$. 
Surfaces of constant probability density 25,26 are also included in all parts of Figure 1 but are not always visible depending on the scale used. Such a surface encloses the given percentage, here 95\%, of the population as estimated via the applicable sample measured. If we instead plot, as in Figure 2a, the curvatures rather than powers for the same eye then we find two clusters of measurements above the label, 100I. The vector space in Figure 2a is not symmetric dioptric power space (as for Figure 1a) and is referred to as surface curvature space $4,6,7$ and, for example, the origin being the null matrix represents a flat surface. Each point in this space represents a specific surface curvature (or shape) by means of a $2 \times 2$ curvature matrix $\mathcal{K}$ (mathematically similar to the $2 \times 2$ dioptric power matrix, F). The axis with label 100 I is the axis of spherical curvatures ${ }^{7}$; that is, it is an infinitely long axis that includes all possible spheres. The other two axes in Figure 2a with labels $100 \mathrm{~J}$ and $100 \mathrm{~K}$ are known as the ortho-antispherical and oblique antispherical axes ${ }^{7}$. These axes represent surfaces that are saddle-shaped with the orientation different for the two axes. For any point on the orthoantispherical axis the principal curvatures are always horizontal and vertical while for the oblique antispherical axis the principal curvatures are always oblique at 45 and $135^{\circ}$. So any three-dimensional surface, such as a corneal surface, can be described as consisting of three separate parts or curvatures, namely a spherical part or curvature and two anti-spherical parts. Consequently any corneal curvature can thus be indicated by a single point in surface curvature space that represents a curvature matrix $\mathcal{K}$ that is effectively the sum of three components, namely $\mathcal{K}_{\mathrm{I}} \mathbf{I}, \mathcal{K}_{\mathrm{J}} \mathbf{J}$ and $\mathcal{K}_{\mathrm{K}} \mathcal{K}$. The spherical and anti-spherical coefficients can be positive or negative. In the upper stereo-pair of Figure $2 \mathrm{a}$ the higher cluster for the posterior surface is slightly larger (that is, measurements are more widely spread) and thus the posterior surface curvatures are more variable than the anterior curvatures. In the lower stereo-pair of Figure 2a the same measurements or clusters (the outermost or black pair) are shown together with two further clusters (the innermost or red pair) that represent the change in curvatures when the original curvatures (in upper stereo-pair of Figure $2 \mathrm{a}$ ) are referred to the mid-corneal surface $S_{m}$. So, the effect of referring the curvatures from the anterior and posterior surfaces to the corneal mid-surface is relatively small but is necessary to provide a common basis for comparison. In (b) and (c) of Figure 2 the scale and origin are adjusted so that we can more easily examine the variation in curvature within each cluster referenced to the corneal mid-surface. The greater variation of the curvatures for the posterior corneal surface when shifted into the mid-surface is obvious when comparing (b) and (c) of Figure 2.

In (d) to (f) of Figure 2 the global and local effects on curvature (which are the crux of this paper) are indicated in the corneal mid-surface of subject 8 . In Figure $2 \mathrm{~d}$ the global and local curvature effects are shown in a single stereo-pair whereas in (e) and (f) of Figure 2 the scale and origin are adjusted so that we can more easily examine the variation in global and local effects on curvature. The sizes and orientations for the surfaces of constant probability density (here $95 \%$ ones) can be used to understand the extent and nature of the local and global effects. The centroids of the ellipsoids in (e) and (f) of Figure 2 are also measures of the means or averages for the global or local effects respectively. For subject 8 , these means are also indicated in Table 2 together with the variances and covariances for the local and global effects. The oblique antispherical variances were largest for both local and global curvature effects and this conforms to the elongation of the ellipsoids mainly along the oblique antispherical axis in (e) and (f) of Figure 2.

A similar analysis (that is, per individual) as above could be used for the other nine subjects but instead in Figure 3a twenty $95 \%$ surfaces of constant probability density are shown for the curvatures of the anterior and posterior corneal surfaces of the 10 subjects after referring curvatures to their corneal mid-surfaces. The ten ellipsoids for curvatures for the posterior corneal surfaces of the ten subjects are indicated towards the top of the stereo-pair in Figure 3a and are seen to be located in a similar part of surface curvature space and some ellipsoids even overlap one another, although sizes, orientations and centroids vary across the different eyes. In Figure $3 \mathrm{~b}$ mean curvatures for the anterior and posterior corneal surfaces for the 10 eyes are indicated, after reference to each of the relevant mid-corneal surfaces, using red dots only, or red dots with $95 \%$ confidence ellipsoids (below). The means are slightly more variable for the posterior surfaces (that is, the upper cluster of dots is more widely spread). 
Figure 4 is perhaps the more crucial figure since it includes $95 \%$ surfaces of constant probability density for the global and local effects in the different corneal mid-surfaces of the ten eyes. In Figure 4a both mean global and local effects for the 10 eyes are included and two small clusters of black dots are seen with the upper cluster for the global effects more widely spread; thus the global effects are slightly more variable and predominate (they are larger in magnitude).

Table 2. For simplicity, vectorized means, $\left(\kappa_{11} \kappa_{21} \kappa_{22}\right)^{\prime}$ from $2 \times 2$ symmetric matrices $\left(\kappa_{21}=\kappa_{12}\right)$ for both the global $\left(\bar{\kappa}_{m}\right)$ and local $\left(\bar{\kappa}_{d}\right)$ mean effects in the corneal mid-surface are provided for the healthy corneas of ten young subjects (S1 to S10 below). Also indicated are vectorized variance-covariances $\left(S_{\mathrm{II}} S_{\mathrm{JJ}} S_{\mathrm{KK}} S_{\mathrm{IJ}} S_{\mathrm{IK}} S_{\mathrm{IK}}\right)^{\prime}$, for the global $\left(\mathbf{S}_{m}\right)$ and local $\left(\mathbf{S}_{d}\right)$ curvature effects in the corneal mid-surfaces for each of the 10 eyes. The matrices are symmetric (for comparative purposes, see similar matrices for the global and local means and variance-covariances in the lower section of Table 1 for the keratoconic eye from Harris and Gillan ${ }^{7}$ ) and thus only the distinct entries (either three or six for the means and variance-covariance matrices respectively) are included here.

\begin{tabular}{|c|c|c|c|c|c|c|c|}
\hline \multirow[b]{2}{*}{$\mathrm{S} 1$} & \multirow[b]{2}{*}{ Global Effects } & Means (D) & \multicolumn{5}{|c|}{ Variances and Covariances $\left(\mathrm{D}^{2}\right)$} \\
\hline & & $\left(\begin{array}{lll}136.75 & 0.37 & 141.42\end{array}\right)^{\prime}$ & $\left(\begin{array}{ll}0.25 & 0.22\end{array}\right.$ & 0.15 & -0.06 & -0.04 & $0.02)^{\prime}$ \\
\hline \multirow{3}{*}{$\mathrm{S} 2$} & Local Effects & $\left(\begin{array}{lll}4.80 & 0.28 & 8.07\end{array}\right)^{\prime}$ & 0.11 & 0.10 & -0.04 & -0.03 & $0.01)^{\prime}$ \\
\hline & Global Effects & $\left(\begin{array}{lll}139.39 & -1.01 & 145.28\end{array}\right)^{\prime}$ & $\left(\begin{array}{ll}0.58 & 0.29\end{array}\right.$ & 0.28 & -0.29 & -0.22 & $0.14)^{\prime}$ \\
\hline & Local Effects & $\left(\begin{array}{lll}6.40 & -0.15 & 9.70\end{array}\right)^{\prime}$ & $\left(\begin{array}{lll}0.67 & 0.38\end{array}\right.$ & 0.34 & -0.36 & $-0.28 \quad 0$ & $0.15)^{\prime}$ \\
\hline \multirow[t]{2}{*}{$\mathrm{S} 3$} & Global Effects & $\left(\begin{array}{lll}138.27 & -0.02 & 140.94\end{array}\right)^{\prime}$ & $\left(\begin{array}{ll}0.64 & 0.74\end{array}\right.$ & 0.62 & $0.00-$ & -0.190 .1 & $.10)^{\prime}$ \\
\hline & Local Effects & $\left(\begin{array}{lll}7.15 & 0.26 & 9.26\end{array}\right)^{\prime}$ & $\left(\begin{array}{lll}0.44 & 1.35\end{array}\right.$ & 0.47 & -0.46 & -0.05 & $-0.45)^{\prime}$ \\
\hline \multirow[t]{2}{*}{ S4 } & Global Effects & $\left(\begin{array}{lll}135.64 & -0.02 & 140.29\end{array}\right)^{\prime}$ & $\left(\begin{array}{ll}0.19 & 0.33\end{array}\right.$ & 0.30 & -0.10 & $0.01-c$ & $0.12)^{\prime}$ \\
\hline & Local Effects & $\left(\begin{array}{lll}7.58 & 0.19 & 9.86\end{array}\right)^{\prime}$ & $\left(\begin{array}{ll}0.27 & 0.38\end{array}\right.$ & 0.29 & -0.11 & -0.07 & $-0.17)^{\prime}$ \\
\hline \multirow[t]{2}{*}{ S5 } & Global Effects & $\left(\begin{array}{lll}140.07 & -0.21 & 144.26\end{array}\right)^{\prime}$ & $\left(\begin{array}{ll}0.21 & 0.24\end{array}\right.$ & 0.40 & -0.17 & -0.17 & $0.07)^{\prime}$ \\
\hline & Local Effects & $\left(\begin{array}{lll}7.43 & 0.08 & 10.78\end{array}\right)^{\prime}$ & $\left(\begin{array}{ll}0.19 & 0.24\end{array}\right.$ & 0.35 & -0.12 & -0.10 & $0.13)^{\prime}$ \\
\hline \multirow[t]{2}{*}{ S6 } & Global Effects & $\left(\begin{array}{lll}141.91 & -1.47 & 142.09\end{array}\right)^{\prime}$ & $(0.78$ & 0.40 & $0.43-$ & $-0.42-$ & $-0.28)^{\prime}$ \\
\hline & Local Effects & $\left(\begin{array}{lll}7.25 & 0.14 & 10.01\end{array}\right)^{\prime}$ & $\left(\begin{array}{ll}0.88 & 0.50\end{array}\right.$ & 0.46 & $0.51-$ & $-0.44-(-1-2$ & $-0.31)^{\prime}$ \\
\hline \multirow[t]{2}{*}{ S7 } & Global Effects & $\left(\begin{array}{lll}140.92 & -0.55 & 144.45\end{array}\right)^{\prime}$ & $(0.22$ & 0.28 & -0.02 & -0.13 & $-0.09)^{\prime}$ \\
\hline & Local Effects & $\left(\begin{array}{lll}7.28 & -0.12 & 10.45\end{array}\right)^{\prime}$ & $(0.32$ & 0.15 & -0.02 & -0.07 & $-0.07)^{\prime}$ \\
\hline \multirow[t]{2}{*}{ S8 } & Global Effects & $\left(\begin{array}{lll}139.00 & -0.78 & 145.90\end{array}\right)^{\prime}$ & $(0.13 \quad 0.14$ & 0.28 & -0.01 & -0.08 & $0.05)^{\prime}$ \\
\hline & Local Effects & $\left(\begin{array}{lll}7.02 & -0.48 & 10.00\end{array}\right)^{\prime}$ & $(0.14 \quad 0.16$ & 0.37 & $0.01-$ & $-0.11-$ & $-0.00)^{\prime}$ \\
\hline \multirow[t]{2}{*}{ S9 } & Global Effects & $\left(\begin{array}{lll}133.25 & -0.86 & 139.85\end{array}\right)^{\prime}$ & $\left(\begin{array}{ll}0.22 & 0.19\end{array}\right.$ & 0.10 & -0.04 & -0.05 & $0.00)^{\prime}$ \\
\hline & Local Effects & $\left(\begin{array}{lll}6.06 & -0.48 & 8.60\end{array}\right)^{\prime}$ & $\left(\begin{array}{ll}0.12 & 0.12\end{array}\right.$ & 0.10 & -0.02 & -0.02 & $0.00)^{\prime}$ \\
\hline \multirow[t]{2}{*}{ S10 } & Global Effects & $\left(\begin{array}{lll}138.75 & 0.68 & 142.01\end{array}\right)^{\prime}$ & $\left(\begin{array}{ll}0.44 & 0.84\end{array}\right.$ & 0.20 & -0.04 & -0.11 & $0.08)^{\prime}$ \\
\hline & Local Effects & $\left(\begin{array}{lll}6.57 & 0.23 & 8.72\end{array}\right)^{\prime}$ & $\left(\begin{array}{ll}1.00 & 0.50 \\
\end{array}\right.$ & 0.45 & -0.35 & $0.42-$ & $-0.26)^{\prime}$ \\
\hline \multirow[t]{2}{*}{ MEANS } & Global Effects & $\left(\begin{array}{lll}138.40 & -0.39 & 142.65\end{array}\right)^{\prime}$ & $\left(\begin{array}{ll}0.37 & 0.36\end{array}\right.$ & 0.30 & -0.03 & $3-0.14$ & $4-0.00)^{\prime}$ \\
\hline & Local Effects & $\left(\begin{array}{lll}6.75 & -0.01 & 9.54\end{array}\right)^{\prime}$ & $\left(\begin{array}{ll}0.43 & 0.40\end{array}\right.$ & 0.31 & -0.10 & -0.06 & $-0.10)^{\prime}$ \\
\hline
\end{tabular}


a)

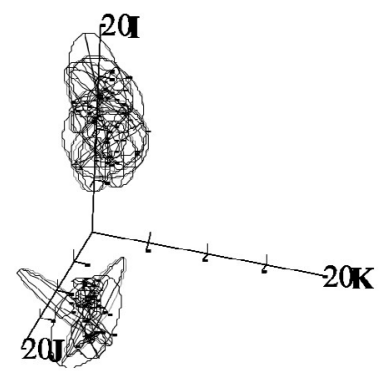

b)

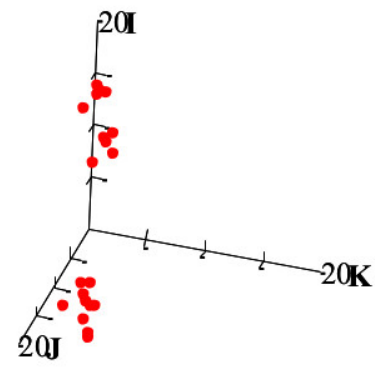

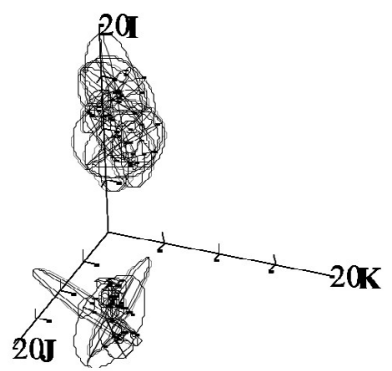

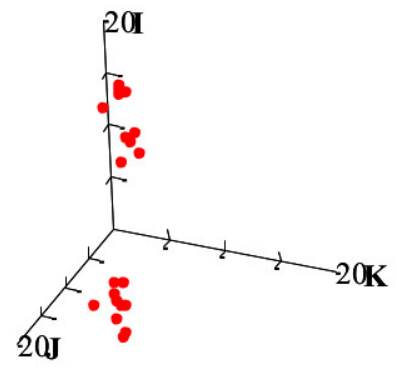

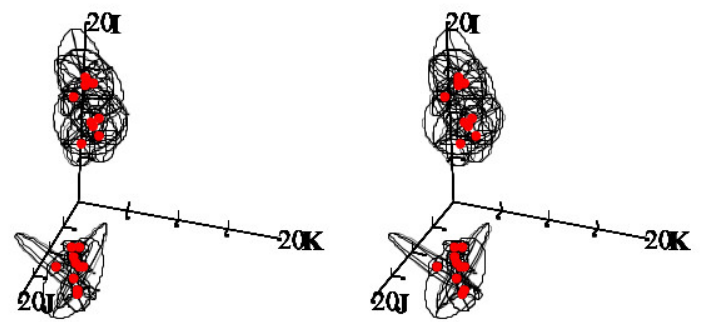

Figure 3. a) Stereo-pair plot of $95 \%$ surfaces of constant probability density for variation in curvature of the anterior and posterior corneal surfaces of all subjects (numbers 1 to 10) are indicated after referring all curvatures to the mid-corneal surfaces of the eyes concerned. Twenty ellipsoids are included (two for each eye) and the upper cluster of ten ellipsoids refers to the posterior corneal surfaces while the lower ellipsoids are for the curvatures of the anterior surfaces. Ellipsoids for Subject 8 (as illustrated in Figures 1 and 2) are also included here. The origin is the curvature 140I D. b) Stereo-pair plots of mean curvatures (red dots) for variation in curvature of the anterior (upper dots and ellipsoids in (a)) and posterior corneal surfaces of the ten eyes are indicated with corresponding $95 \%$ surfaces of constant probability density for the means (lower stereo-pair). These ellipsoids inform us about variation in mean curvature rather than variation in the populations of raw curvatures (the ellipsoids in Figure 3a). The origin is the curvature $10 \mathbf{I} \mathrm{D}$.

The cluster of dots for the local effects is seen at the origin, the null matrix. So the local effects are small in magnitude. Both clusters also have $95 \%$ surfaces of constant probability density but these are not readily visible at the scale used. In Figure $4 \mathrm{~b}$ the global means (the upper cluster of dots in Figure 4a) are again shown but with red dots and 95\% confidence ellipsoids for the respective means concerned. The ellipsoidal volumes, orientations and locations are slightly different for the various eyes but generally the volumes are small and similar whereas orientations can be markedly different for the various eyes involved. In Figure $4 \mathrm{c}$ the local means (the lower cluster of dots in Figure 4a) are indicated with a different origin and scale so as to again more easily examine the situation. Most of these confidence ellipsoids are small and often overlap one another but again orientations vary quite a lot across the eyes. But, as Harris and Gillan ${ }^{7}$ also noted for their keratoconic eye, the global effects dominate in terms of variation in curvature, and where measurements are considered in the corneal mid-surface. Comparing the global and local effects matrices of Table 1 for the keratoconic cornea $^{7}$ to that of the different healthy corneas in Table 2 or to the means in Table 2 mainly indicates similarities of such effects, with the possible exception of the off-diagonal entries in the matrices. For the healthy corneas the off-diagonal entries $\left(\kappa_{21}=\kappa_{12}\right.$, or the torsional curvature in the reference meridian) were individually and on average generally much smaller than for the keratoconic eye, perhaps relating to the effects of keratoconus on corneal astigmatism. The variances and covariances for the global effects in Tables 1 and 2 are fairly similar but there is some suggestion that possibly for the healthy corneas there is similar variation along the three axes (essentially implying random variation in all directions of curvature space) whereas variation is more profound along the ortho-antispherical axis $\left(\mathcal{K}_{\mathrm{J}} \mathbf{J}\right)$ for the keratoconic cornea ${ }^{7}$ (probably relating to blinking effects on a weakened corneal structure). For the local effects, variation was random or similar in all directions for both the keratoconic ${ }^{7}$ and healthy corneas. Covariances were, individually and on average, small and close to zero for all eyes and indicate little evidence for linear relationships between the different pairs of variances concerned. 


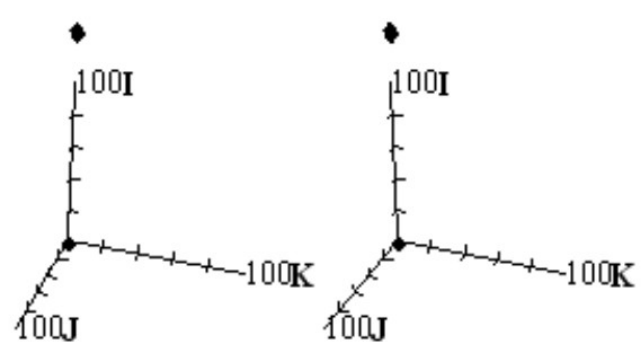

a)
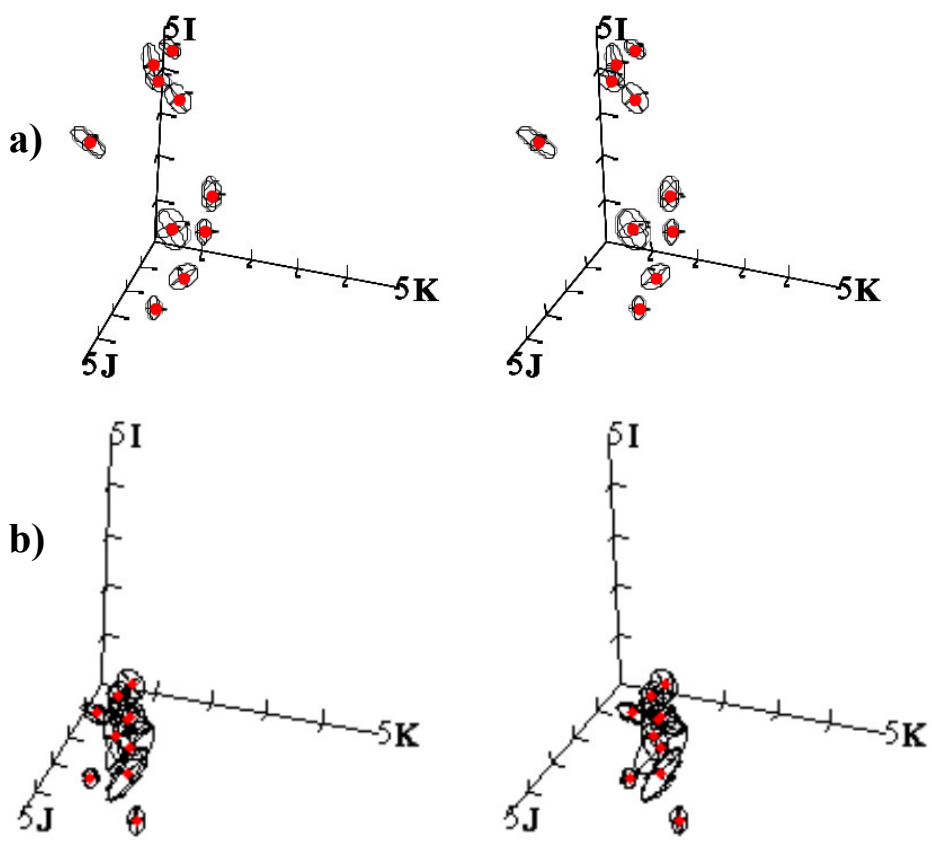

Fjgure 4. a) Stereo-pair plot of the local (the cluster of dots and ellipsoids near the origin) and global (the upper dots and ellipsoids) effects for variation in curvature of the corneal surfaces of the 10 eyes are included. b) The $95 \%$ confidence ellipsoids and mean global effects for the 10 eyes after referring to the respective mid-corneal planes are shown. The origin is the curvature 140 I D. c) The $95 \%$ confidence ellipsoids and mean local effects for the 10 eyes after referring to the respective midcorneal planes are shown. The origin is the curvature 10 I D.

\section{Discussion}

As mentioned earlier anatomically and physiologically the human cornea is not a simple structure and one can consider variation of human corneas in various ways. This papermainly concentrates on variation in surface curvature (essentially the inverse of the surface radius and, unlike for power, this quantity is independent of refractive indices surrounding the surface) of both the anterior and posterior surfaces as investigated using a simple model ${ }^{4}$ that refers such variation to the corneal mid-surface. This reduces the impact or influence of corneal thickness since the changes are referred to the same surface. An alternate approach that is also briefly considered in this paper is variation of the anterior and posterior corneal powers.
(Of course, many other parameters of the cornea also vary but are not of interest here.) So examples of corneal variation in terms of power and curvature are both included in this paper and two vector spaces are involved in studying these different types of variation; namely symmetric dioptric power space and surface curvature space ${ }^{4}, 7$. There are many mathematical commonalities between the two vector spaces and similar quantitative and qualitative approaches can be used for both variables, that is, power or curvature. Both are expressed mathematically ${ }^{4,7}$ using a symmetric $2 \times 2$ matrix $\mathbf{F}$ for power or $\mathcal{K}$ for curvature and understanding variation in either dioptric power or surface curvature requires a $3 \times 3$ variance-covariance matrix, $\mathbf{S}$. Examples of such matrices for both healthy eyes and for an eye with mild keratoconus ${ }^{6,7}$ are included herein. Stereo-pair scatter plots with or without surfaces of constant probability density ${ }^{25,26}$ (including a defined percentage of either the population of (raw) measurements, or defining a region within which one can be confident to a specified level that the sample mean is located) can be readily applied to represent graphically intra- or inter-subjective variation in either power or curvature and again several examples of these plots are included in this paper. The issue of whether one studies variation of power or curvature is crucial and depends upon the purposes of the study itself. Thus power would be the relevant quantity for investigations of optical aspects of the cornea whereas curvature is relevant where one needs to understand the nature and possible causes of variation in change in physical corneal shape, form or topography. This paper mainly concerns the latter although some mention of the former is also included to hopefully facilitate broader understanding of the similarities and differences involved with the two approaches to studying corneal variation.

In this paper variation in corneal curvature is primarily studied in 10 healthy young eyes but for comparative purposes previous results from Harris and Gillan ${ }^{7}$ for a young, mildly keratoconic cornea are also briefly discussed. Thus far these 11 eyes are the only eyes that have been explored using the mathematical model ${ }^{4,7}$ and methods ${ }^{4,7}$ contained herein. The results were similar in some respects for both the healthy and keratoconic eyes. For instance, for both types of corneas the global or larger-scale effects dominate the local or smaller-scale ones and these global effects indicate the extent to which the anterior and posterior corneal 
surfaces or curvatures of an eye vary in such a manner that the two surfaces are similar to one another. The global effects are measures of the similarity or matching of the anterior and posterior surface curvatures and they are each a semi-sum or (arithmetic) mean of the two curvatures (see equation 3 ) referred to the corneal mid-surface 6 . Since they vary over time we can define a mean global effect for a cornea of an eye. Similarly, local effects are measures of the dissimilarity, differences or extent of departure from matching of the anterior and posterior surface curvatures and they are each a semidifference of the two curvatures (see equation 4), again referred to the corneal mid-surface ${ }^{6}$. So a local effect in the mid-surface provides an indication of the extent to which the relevant curvatures differ and they are measures of independence of the two corneal surfaces ${ }^{4}$. Again, it is possible to define an average or mean local effect provided that multiple measurements of the relevant quantities (anterior and posterior curvatures and axial corneal thickness) are obtained over either a short- or long-term period of time. This paper concerns measurements that were obtained over about a one-hour period for each of the eyes involved and so it mainly involves short-term variation in corneal curvatures and axial or central thicknesses. Many factors might influence global or local curvature effects and could include the processes of lid blinking, tear evaporation, ocular movements, heterogeneity of tear or corneal components, the intraocular pressure or effects of lens accommodation and vascular pulses and heart rate on the cornea, et cetera. Corneal oedema, inflammation or other disorders such as keratoconus are known to have important influences on corneal curvatures that are likely to be amenable to study using the model ${ }^{4}$ as used in this paper. The methods will also assist towards understanding the nature of approaches used towards measurement of corneas, such as keratometry or corneal topography and here specifically some parameters as determined with Scheimpflug scanning photography of the cornea were investigated in detail.

No attempt has been made in this study to align or centre the different corneal maps $(N=43)$ for each of the individuals $(N=10)$ concerned and this would naturally contribute to some extent towards the short-term variation in the anterior and posterior surfaces. The issue of measurement of the posterior corneal surface through that of the air-tear interface or anterior corneal surface similarly is another important source of uncertainty, noise or variation in the measurements of the posterior corneal surface. But, the purpose of this paper was to illustrate a method of analysis of variation of corneal powers versus corneal curvatures and not to specifically investigate the relative contributions of different sources to such variation, whether due the abovementioned issues or to micro-fluctuations 28 of the surfaces or any other factors such as stability of the tears after blinking 29 or possible corneal affects of ocular accommodation ${ }^{30}$. Nonetheless, many of these issues would be very productive areas for future study with some of the ideas in references 4 and 7, and also herein, and with methods as used by Buehrens et al in their papers ${ }^{28-30}$.

The terminology of surface curvature space, and of the three coordinate axes themselves, may be a little unfamiliar or perhaps even slightly intimidating at first but they are important towards more completely understanding the nature and causes of physical changes or variation of the cornea. The shape, orientation and size of ellipsoids, and of the corresponding spread or distribution of data, provide important or vital clues as to the underlying processes that may affect a specific cornea at any moment of time or over a period of time and thus this model has potential applications towards fuller understanding, and possibly even improving, various types of refractive surgery involving the cornea, or of properly evaluating treatments such as collagen cross-linking or keratoplasty that might be used in certain corneal disorders.

Future studies could use curvatures to investigate, for example, ageing effects on the human cornea. Similarly, studies of corneal changes in pregnancy or relating to menstrual cycles or diabetes might be quite informative. A repeatability study in a larger sample of, say, 500 eyes, where only two sets of measurements per eye for the anterior and posterior corneal curvatures and axial thicknesses remains a promising topic for the future.

The mathematical model ${ }^{4}$ used here, although relatively basic, could perhaps be further developed to enhance its possible applications and overall usefulness. Presently it is a linear model applied only to the central or apical parts of the two corneal surfaces and axial thickness but perhaps it could be modified for use for data obtained from the entire anterior and posterior corneal surfaces and their corresponding or related thicknesses. But, the model ${ }^{4}$ in its current state does provide a unique and relatively simple and useful method for studying possibly the main or most profound 
aspects that affect corneal function and indirectly vision function and performance.

\section{Acknowledgements}

To the subjects who kindly allowed us to obtain the relevant measurements for this paper and to Professor WF Harris for discussions and advice.

\section{References}

1. Vasudevan B, Simpson TL, Sivak JG. Regional variation in the refractive index of the bovine and human cornea. Optom Vis Sci 200885 977-981.

2. Mejía-Barbosa Y, Malavara-Hernández D. A review of methods for measuring corneal topography. Optom Vis Sci 200178 240-253.

3. Cronje-Dunn S. Short-term keratometric variation in the human eye. Masters dissertation in the Department of Optometry, Rand Afrikaans University, South Africa, 1995.

4. Harris WF. Global and local contributions to corneal surface curvature: a simple model. S Afr Optom 200867 11-16.

5. Mathebula SD. Quantitative analysis of the linear optical character of the anterior segment of the eye. Doctoral thesis in the Department of Optometry, University of Johannesburg, South Africa, 2010.

6. Gillan WDH. Variation in surface power and thickness of a moderately keratoconic cornea. S Afr Optom 200867 4-10.

7. Harris WF, Gillan WDH. Global and local contributions to corneal surface curvature of a moderately keratoconic cornea. S Afr Optom 200867 17-20.

8. Read SA, Collins MJ. Diurnal variation of corneal shape and thickness. Optom Vis Sci 200986 170-180.

9. Twa MD, Parthasarathy S, Roberts C, Mahmoud AM, Raasch TW, Bullimore MA. Automated decision tree classification of corneal shape. Optom Vis Sci 200582 1038-1046.

10. Shirayama M, Wang L, Weikert MP, Koch DD. Comparison of corneal powers obtained from 4 different devices. $\mathrm{Am} \mathrm{J}$ Ophthalmol 2009148 528-535.

11. Potvin R, Fonn D, Sorbara L. In vivo comparison of corneal topography and keratometry systems. ICLC 199623 20-25.

12. Mutti DO, Mitchell L, Jones LA, Friedman NE, Frane SL, Lin WK, Moeschberger ML, Zadnik K. Refractive astigmatism and the toricity of ocular components in human infants. Optom Vis Sci 200481 753-761.

13. Remón L, Benlloch J, Furlan WD. Corneal and refractive astigmatism in adults: a power vectors analysis. Optom Vis Sci 200986 753-761.

14. Kounis GA, Tsilimbaris MK, Kymionis GD, Ginis HS, Pallikaris IG. Estimating variability in Placido-based topographic systems. Optom Vis Sci 200784 E962-E968.

15. Braaf B, Dubbelman M, van der Heijde RGL, Sicam VADP. Performance in specular reflection and self-imaging corneal topography. Optom Vis Sci 200986 467-475.
16. Giráldez MJ, Yebra-Pimentel E, Parafita MA, Escandón S, Cerviño A, Pérez MV. Comparison of keratometric values of healthy eyes measured by Javal keratometer, Nidek autokeratometer, and corneal analysis system (EyeSys). ICLC 200027 33-38.

17. Owens H, Garner LF, Craig JP, Gamble G. Posterior corneal changes with orthokeratology. Optom Vis Sci 200481 421426.

18. Martin R, Izquierdo M, Saber A. Investigation of posterior corneal curvature in CL-induced corneal swelling. Contact Lens Anterior Eye 200932 288-293.

19. Blendowske R. Hans-Heinrich Fick: early contributions to the theory of astigmatic systems. S Afr Optom $200362105-$ 110 .

20. Long WF. A matrix formalism for decentration problems. Am J Optom Physiol Opt 197653 27-33.

21. Mathebula SD, Rubin A, Harris WF. Quantitative analysis in Hamiltonian space of the transformed ray transferences of a cornea. S Afr Optom 200766 68-76.

22. Mathebula SD, Rubin A. A comparative study of autokeratometric and Scheimpflug keratometric measurements of the anterior corneal surface: results for a single subject. $S$ Afr Optom 200766 109-119.

23. Mathebula SD, Rubin A. Short-term variation of central corneal thickness and axial anterior chamber depth of healthy eyes using Scheimpflug photography via the Oculus Pentacam. S Afr Optom 200968 12-24.

24. Thibos LN, Wheeler W and Horner D. Power vectors: an application of Fourier analysis to the description and statistical analysis of refractive error. Optom Vis Sci 199774 367-375.

25. Harris WF, Malan DJ, Rubin A. The distribution of dioptric power: ellipsoids of constant probability density. Ophthalmic Physiol Opt 199111 381-384.

26. Harris WF, Malan DJ, Rubin A. Ellipsoidal confidence regions for mean refractive state. Optom Vis Sci 199168950 953.

27. Harris WF. Interconverting the matrix and principal meridional representations of dioptric power in general including powers with nonorthogonal and complex principal meridians. Ophthalmic Physiol Opt 200121 247-252.

28. Buerhen T, Lee B, Collins MJ, Iskander DR. Ocular microfluctuations and videokeratoscopy. Cornea $200221346-$ 351.

29. Buerhen T, Collins MJ, Iskander DR, Davis B, Lingelbach B. The stability of corneal topography in the post-blink interval. Cornea 200120 826-833.

30. Buerhen T, Collins MJ, Loughridge J, Carney LG, Iskander DR. Corneal topography and accommodation. Cornea 2003 22 311-316. 


\section{Appendix}

The first measurement (of a sample of 43 values) for each of the anterior and posterior corneal curvatures and axial corneal thicknesses for the right eye of Subject 8 is used here to illustrate the simple mathematical model ${ }^{4}$ as applied in this paper:

The first measurement of corneal thickness was 526 micrometres or $0.526 \mathrm{~mm}$. The first measurement for the two principal meridians of the anterior corneal surface was $7.86 \mathrm{~mm}$ along $14.2^{\circ}$ and 7.60 along $104.2^{\circ}$ Similarly, the first measurement for the two principal meridians of the posterior corneal surface was $6.66 \mathrm{~mm}$ along $5.3^{\circ}$ and 6.17 along $95.3^{\circ}$ Each of the four radii are simply inverted (for example, for the largest principal radius of the anterior surface, that is, $7.86 \mathrm{~mm}$ along $14.2^{\circ}$ we have $\mathcal{K}_{1}=\frac{1}{0.00786 \mathrm{~m}}=127.23 \mathrm{D}$ ) to produce the two curvatures along the principal meridians. The anterior surface of this cornea expressed as curvatures is $\mathcal{K}_{1}\left\{\mathrm{~A}_{1}\right\} \mathcal{K}_{2}\left\{\mathrm{~A}_{2}\right\}$, or $127.23 \mathrm{D}\left\{14.2^{\circ}\right\} 131.58 \mathrm{D}\left\{104.2^{\circ}\right\}$. This notation is described as principal meridional notation $^{27}$. The three equations ${ }^{27}$ below (solving the reverse eigenvalue problem ${ }^{4}, 27$ ) are then used to determine the entries in the curvature matrices for the anterior and posterior corneal surfaces:

$\kappa_{11}=\mathcal{K}_{1} \cos ^{2} A_{1}+\mathcal{K}_{2} \cos ^{2} A_{2}$,

$\kappa_{12}=\kappa_{21}=\mathcal{K}_{1} \sin A_{1} \cos A_{1}+\mathcal{K}_{2} \sin A_{2} \cos A_{2}$

and

$\kappa_{22}=\mathcal{K}_{1} \sin ^{2} A_{1}+\mathcal{K}_{2} \sin ^{2} A_{2}$.

The anterior and posterior curvature matrices are respectively

$\mathcal{K}_{1}=\left(\begin{array}{ll}\kappa_{11} & \kappa_{12} \\ \kappa_{21} & \kappa_{22}\end{array}\right)=\left(\begin{array}{cc}127.49 & -1.04 \\ -1.04 & 131.32\end{array}\right)$ D and $\mathcal{K}_{2}=\left(\begin{array}{cc}150.25 & -1.10 \\ -1.10 & 161.97\end{array}\right)$ D. Referred to the mid-corneal surface (using Equations 5 and 6) these matrices become $\mathcal{K}_{1 m}=\left(\begin{array}{cc}131.91 & -1.11 \\ -1.11 & 136.01\end{array}\right) \mathrm{D}$ and $\mathcal{K}_{2 m}=\left(\begin{array}{cc}144.54 & -1.01 \\ -1.01 & 155.35\end{array}\right) \mathrm{D}$ respectively. Thereafter, with Equations 3 and 4 the global and local effects respectively are $\mathcal{K}_{m}=\left(\begin{array}{cc}138.23 & -1.06 \\ -1.06 & 145.68\end{array}\right) \mathrm{D}$ and $\mathcal{K}_{d 2}=\left(\begin{array}{ll}6.31 & 0.05 \\ 0.05 & 9.67\end{array}\right) \mathrm{D}$. The global effects dominate, that is, have larger magnitudes.

For this cornea, the same approach as above is then used with each of the subsequent 42 measurements of these three parameters (namely anterior and posterior corneal curvatures, and axial corneal thickness) and the mean global and local effects are then calculated using simple arithmetic averages.

To represent all the measurements ( $N=43$ for this right eye) in surface curvature space Equations 7-9 are used (to convert to the appropriate coordinates used in the space) with either the curvature matrices ( $\mathcal{K}_{1 i}$ and $\mathcal{K}_{2 i}$ where subscript $i=1,2 \ldots 43$ ) themselves (remembering that in this context there are 43 of each type of these matrices) or the same matrices when shifted to the mid-corneal surface ( $\mathcal{K}_{m i}$ and $\mathcal{K}_{2 m i}$ and , $\left.i=1,2 \ldots 43\right)$ or with the global $\left(\mathcal{K}_{m i}, i=1,2 \ldots 43\right)$ and local $\left(\mathcal{K}_{d 2 i}, i=1,2 \ldots 43\right)$ effects matrices. In this paper, examples for Subject 8 in isolation (Figure 2) and for all 10 subjects together (Figures 3 and 4) are provided. 